\title{
ReMed:
}

\section{Krisen haben viele Gesichter}

\section{Iris Leu, Peter Christen}

Für den Leitungsausschuss ReMed

Krisen machen auch vor Ärztinnen und Ärzten nicht Halt. Unvermittelt durchbrechen sie den Alltag und stellen das Leben auf den Kopf - ein Konflikt bei der Arbeit, der plötzlich ausartet, ein Behandlungsfehler, der das Selbstvertrauen erschüttert, oder Beziehungsprobleme, die depressive Verstimmungen auslösen.

ReMed bietet in solchen kritischen Momenten rasch und unbürokratisch Hilfe. Bei jeder Kontaktaufnahme meldet sich ein erfahrenes Beraterteam innerhalb von 72 Stunden und bespricht die persönliche Situation.

Burnout macht auch vor Ärztinnen und Ärzten nicht Halt, im Gegenteil, sie sind besonders gefährdet, in eine solche Krise zu fallen. Mindestens $20 \%$ der Ärzte leiden an einem Burnout-Syndrom, 78\% sind unzufrieden mit ihrem Beruf. Burnout ist kein sporadisches Phänomen mehr, sondern hat sich längst zu einem Flächenbrand im Gesundheitswesen entwickelt, sagt Dr. med. Thomas Bergner, Facharzt in eigener Praxis und Autor des Buches Burnout bei Ärzten.

Doch die Vielfältigkeit der krankmachenden Faktoren und die Vielzahl der Symptome erschweren es,

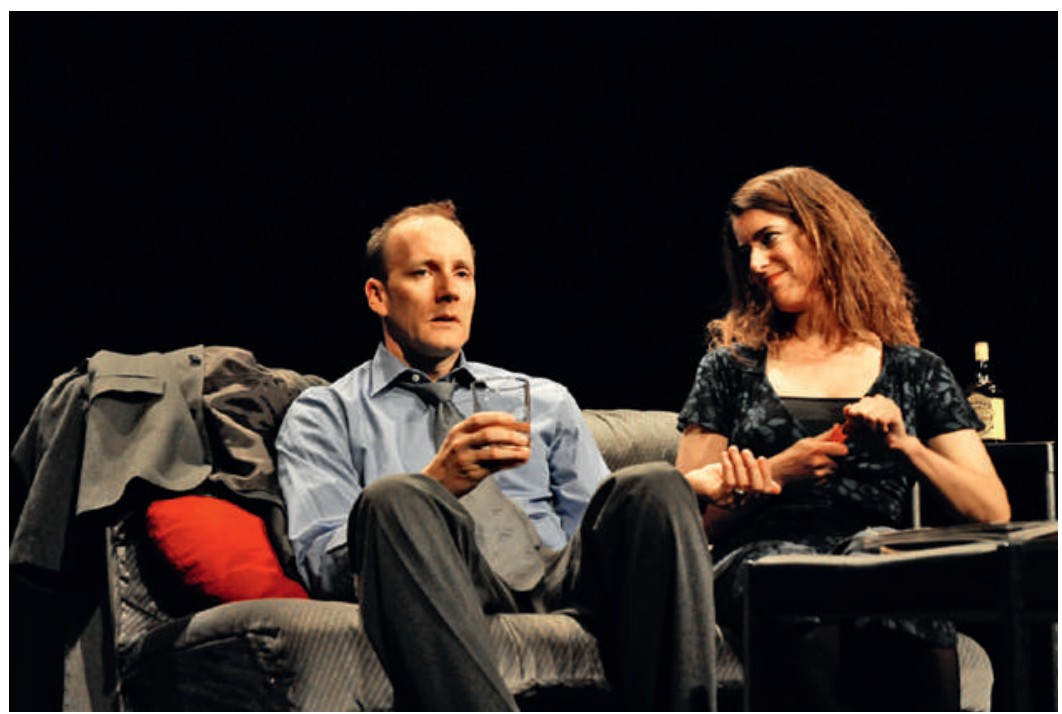

Der vermehrte Griff zum Alkohol: auch ein Zeichen von Erschöpfung, Überforderung und Burnout.
Burnout rechtzeitig zu erkennen. ReMed, das Unterstützungsnetzwerk für Ärztinnen und Ärzte, hat diese Schwierigkeit erkannt und wirkt neben den Beratungen mit seinem Angebot auch präventiv.

\section{Interaktives Theater}

Eine Möglichkeit zur Prävention und Sensibilisierung bieten Workshops und Weiterbildungen unter Einbezug von interaktivem Theater. Die Zuschauer werden durch gezielte Moderation animiert, die Handlungsweisen der Schauspielerinnen und Schauspieler nach den eigenen Vorstellungen zu gestalten. Durch das aktive Eingreifen des Publikums werden

Das Theater bietet Zuschauern die Möglichkeit, in eine bekannte Rolle zu schlüpfen und gemeinsam nach Lösungen zu suchen.

die eigene Sicht der Dinge direkt ausprobiert und die Folgen des eigenen Handelns unmittelbar gespürt.

Dieses Angebot, das ein nachhaltiges Training für zukünftiges Handeln in Konfliktsituationen ist, richtet sich an die kantonalen Ärztegesellschaften, medizinischen Fachgesellschaften bzw. an alle Ärzteorganisationen, an soziale Institutionen, an medizinische Bildungseinrichtungen, an Unternehmen und auch an die breite Öffentlichkeit.

Sowohl in der Deutschschweiz wie auch in der Romandie fanden in den letzten Wochen/Monaten solche interaktiven Theater in Zusammenarbeit mit ReMed statt. Zwei Erfahrungsberichte

\section{Fortbildung mit Hausärzten im Kantonsspital} Glarus mit dem «Knotenpunkt»

Mit der Inszenierung «Der Nächste bitte» greifen die vier Schauspieler eine Szene aus dem Leben eines Arztes auf. Es wird pointiert vorgeführt, wie schleichend und von den Betroffenen und den Angehörigen vorerst unbemerkt das Fass langsam überläuft.

- Die Praxis fordert alles.

- Die Familie bekommt nichts.

- Die Freunde ziehen sich zurück. 
Burnout ist eine Form von Krise. Der Arzt, der in der Praxis kaum mehr Geduld für die Anliegen seiner Patienten aufbringen kann, der zu Hause mit seiner Frau nur noch schweigen mag und keine Energie mehr hat, seiner Tochter eine Geschichte zu erzählen. Derselbe Arzt zieht sich von seinen Freunden zurück und greift immer öfter zum Alkohol.

Mit diesem Stück wird dem Publikum aufgezeigt, wann und wie das Umfeld reagieren kann und soll und wann ein «Stopp» nötig wird, zum Schutz des Betroffenen. Mit «Stopp» unterbricht Fra Zeller, die Moderatorin, die Schauspieler und fordert die $\mathrm{Zu}$ -

\section{Die Gruppe verstand es bestens, professionell und witzig die Anwesenden über ihr eigenes Burnout reflektieren zu lassen.}

schauer auf, ihre Lösungsvorschläge direkt selber zu spielen. Diese Art von Theater bietet dem Publikum die Möglichkeit, in eine ihnen oft bekannte Rolle zu schlüpfen und gemeinsam mit den übrigen Zuschauern nach Lösungen zu suchen, um das Risiko einer solchen Krise zu senken und die Folgen des eigenen Handelns direkt zu spüren.

Die Vorstellung in Glarus war ein voller Erfolg. Die Zuschauer waren begeistert und motiviert, durch das aktive Eingreifen die eigene Sicht der Dinge auszuprobieren und zu erkennen, wie schwierig es oft ist, in einer Krise richtig zu handeln. Der anschliessende Apéro wurde zum Austausch von Erfahrungen und zum Knüpfen von neuen Kontakten genutzt.

Interaktives Theater auf Deutsch: «Knotenpunkt» Krisen haben viele Gesichter - neben Burnout spielt "Knotenpunkt" auch Stücke zu anderen Tabuthemen wie Alkohol oder Depression, Demenz/Alzheimer, Gewalt im Alter, Krebs, Hirnverletzung und Angst/Panik/Phobien.

Zusätzlich bietet «Knotenpunkt» massgeschneiderte Szenen in Form von Coachings an, die akute Probleme aufgreifen.

Bei diesen realitätsnahen Szenen kann experimentiert und interagiert werden. Das Angebot dient als nachhaltiges Training für zukünftiges Handeln in Konfliktsituationen. Es richtet sich an Ärzteorganisationen, an soziale Institutionen, an medizinische Bildungseinrichtungen, an Unternehmen und auch an die breite Öffentlichkeit.

Weitere Informationen sind auf www.theater-knotenpunkt.ch zu finden.
Interaktives Theater auf Französisch: «Le Caméléon» Die Compagnie "Le Caméléon" ist in der Westschweiz seit 20 Jahren aktiv, seit 2012 auch in der Deutschschweiz. Sie setzt sich zusammen aus siebzehn professionellen Schauspielerinnen und Schauspielern aus der Romandie und sieben aus der Deutschschweiz; die meisten verfügen über eine Zweitausbildung im Sozialbereich. Inspiriert wird die von "Le Caméléon» angewandte Methode durch das "Theater der Unterdrückten» nach Augusto Boal. Die Methode des brasilianischen Theatermannes Augusto Boal ermöglicht es, interaktiv und humorvoll schwierige Themen zu behandeln, ohne moralisierenden Unterton. Das Ziel der Compagnie ist, durch interaktive Animation die Ressourcen, die jeder Mensch besitzt, bewusst zu machen. Neben Forum Theater bietet die Compagnie auch Interventionen an Seminaren, Kongressen und an Ausbildungstagen diverser Berufszweige an. Einen Überblick über das Angebot finden Sie auf www.lecameleon.ch.

\section{Forum Theater "Le Caméléon" am Quadrimed- Kongress in Crans-Montana}

Im Theaterstück "Comment allez-vous docteur? Notre quotidien sous la loupe des comédiens de la troupe Caméléon» begleitet das Publikum den Arzt Herbert im Jahr 2048 (!). Zuerst als Assistenzarzt, später in seiner Praxis ist er durch Notfälle, anspruchsvolle Patienten, eine verpasste Diagnose und überbordende Administration heillos überfordert. Er greift zur Whisky-Flasche, betreibt Selbstmedikation mit Psychopharma-Cocktails. Auch Herbert vernachlässigt seine Familie und ist am Ende seiner Kräfte. In der Aufführung des Caméléon-Theaters werden die anwesenden Ärztinnen und Ärzte unter der kompetenten und humorvollen Moderation des «Jokers» Carlos Henriquez ebenfalls in das Stück einbezogen. Sie werden eingeladen, auf der Bühne in die Rolle von Herbert, Kolleginnen und Ehefrau zu schlüpfen, neue Verhaltensweisen auszuprobieren und Wege zu einer neuen Work-Life-Balance zu suchen. Die Gruppe verstand es bestens, professionell und witzig die Anwesenden über ihr eigenes Burnout reflektieren und sich mit den Kollegen austauschen zu lassen.

\section{Kontaktieren Sie ReMed}

Wenn Sie interessiert sind, eine interaktive Veranstaltung in Ihrer Organisation bzw. Institution anzubieten oder als Zuschauer dabei zu sein, melden Sie sich unter info[at]swissremed.ch. 